Pacific Journal of Mathematics

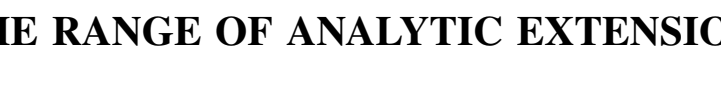




\section{THE RANGE OF ANALYTIC EXTENSIONS}

\section{J. GLobevnik}

Denote by $\Delta, \bar{J}, \partial \Delta$ the open unit dise in $C$, its closure and its boundary, respectively. Let $X$ be a complex Banach space and denote by $\mathscr{A}(X)$ the class of all non-empty sets $P \subset X$ having the following property: given any closed set $F \subset \partial \Delta$ of measure 0 and any continuous function $f: F \rightarrow P$ there exists a continuous extension $\tilde{f:} \bar{J} \rightarrow X$ of $f$, analytic on $\Delta$ and satisfying $\tilde{f}(\bar{\Delta}-F) \subset \operatorname{Int} P$.

Theorem. $P \in \mathscr{A}(X)$ if and only if Int $P$ is connected, locally connected at every point of $P$ and satisfies $P \subset$ closure (Int $P$ ).

TheOREM. If $P \subset C$ consists of more than one point then $P \in \mathscr{A}(C)$ if and only if given any $F$ and $f$ as above there exists a continuous extension $\hat{f}: \bar{\Delta} \rightarrow C$ of $f$, analytic on $\Delta$ and satisfying $\tilde{f}(\bar{\Delta}) \subset P$.

This generalizes a theorem of Rudin which asserts that such $\hat{f}$ exists if $P \subset C$ is homeomorphic to $\bar{A}$.

Theorem. If $P \in \mathscr{A}(X)$ then given any relatively open set $B \subset \partial \Delta$, any relatively closed set $F \subset B$ of measure 0 and any continuous function $f: F \rightarrow P$ there exists a continuous extension $\tilde{f}: \Delta \cup B \rightarrow X$ of $f$, analytic on $\Delta$ and satisfying $\tilde{f}((\Delta \cup B)-F) \subset \operatorname{Int} P$.

o. Introduction. Throughout, we denote by $\Delta, \bar{\Delta}$ and $\partial \Delta$ the open unit disc in $C$, its closure and its boundary, respectively. If $X$ is a complex Banach space and $r>0$ we write $B_{r}(X)=\{x \in X$ : $\|x\|<r\}$. Let $x \in X$ and $S, T \subset X$. We write $x+S=\{x+u: u \in S\}$ and $S+T=\{u+v: u \in S, v \in T\}$. We denote by Int $S, \bar{S}$ the interior of $S$ and the closure of $S$, respectively. If $F$ is a compact Hausdorff space we denote by $C(F, X)$ the set of all continuous functions from $F$ to $X$ and write $C(F)$ for $C(F, C)$. If $B \subset \partial \Delta$ is a relatively open set we denote by $H_{B}(\Delta, X)$ the set of all continuous functions from $\Delta \cup B$ to $X$ which are analytic on $\Delta$. For $H_{\partial \Delta}(\Delta, X)$ we write $A(\Delta, X)$ and for $A(\Delta, C)$, the disc algebra, we write $A(\Delta)$. We denote the set of all positive integers by $N$. If $a, b \in R, a<b$ we write $[a, b]=\{t \in R: a \leqq t \leqq b\}$ and we denote $[O, 1]$ by $I$.

The well known Rudin-Carleson theorem [3, 19, 22] states that given a closed set $F \subset \partial \Delta$ of measure 0 and $f \in C(F)$ there exists an extension $\tilde{f} \in A(\Delta)$ of $f$ satisfying

$$
\max _{z \in \bar{J}}|\widetilde{f}(z)|=\max _{s \in F}|f(s)| \text {. }
$$


Actually the following theorem was proved by Rudin: Given $F$ as above, let $P \subset C$ be homeomorphic to $\bar{\Delta}$ and let $f \in C(F)$ satisfy $f\left(F^{\prime}\right) \subset P$. There exists an extension $\widetilde{f} \in A(\Delta)$ of $f$ satisfying $\widetilde{f}(\bar{\Delta}) \subset P$. An interesting consequence is that given any $P \subset C$ homeomorphic to $\bar{\Delta}$ there exists $\tilde{f} \in A(\Delta)$ satisfying $\tilde{f}(\bar{\Delta})=\tilde{f}(\partial \Delta)=P[2,4]$.

The Rudin-Carleson theorem was generalized into several directions. A consequence of its generalization to the functions into a Banach space $[21,18,7]$ is that for any finite-dimensional complex normed space $X$ there exists $\tilde{f} \in A(\Delta, X)$ such that $\tilde{f}(\bar{J})=\overline{B_{1}(X)}$ [7]. Heard and Wells [12] generalized the Rudin-Carleson theorem as follows: Let $B \subset \partial \Delta$ be a relatively open set and $F \subset B$ a relatively closed set of measure 0 . Given any bounded continuous function $f: F \rightarrow C$ there exists an extension $\tilde{f} \in H_{B}(\Delta, C)$ of $f$ satisfying

$$
\sup _{z \in \Delta}|\tilde{f}(z)|=\sup _{s \in F}|f(s)| \text {. }
$$

The generalization of this result to the functions into a Banach space $X$ [8] makes possible, in the case when $X$ is separable, to prove the existence of a continuous function $\widetilde{f}: \bar{A}-\{1\} \rightarrow X$, analytic on $\Delta$, whose range is contained and dense in $\overline{B_{1}(X)}$ and whose cluster set at 1 is $\overline{B_{1}(X)}$ [8].

The applications above seem interesting enough to consider the following general problem.

Problem. Let $X$ be a complex Banach space, $B \subset \partial \Delta$ a relatively open set, $F \subset B$ a relatively closed set of measure 0 and let $f: F \rightarrow X$ be a continuous function. Assume that a subset $P$ of $X$ contains $f(F)$. Under what conditions on $P$ does there exist an extension $\widetilde{f} \in H_{B}(\Delta, X)$ of $f$ satisfying $\widetilde{f}(\Delta \cup B) \subset P$ ?

By the results mentioned above such an $\tilde{f}$ exists if $P \subset X$ is a closed ball. To prove this one needs the fact that the subspace of all bounded functions in $H_{B}(\Delta, X)$ is a left $A(\Delta)$-module and the fact that $P$ is absolutely convex in order to make the necessary norm estimations on the interpolating function $\tilde{f}$ assuring that $\tilde{f}(\Delta \cup B) \subset P$ [8]. Nothing similar is true in general when we consider the functions in $H_{B}(\Delta, X)$ whose ranges are contained in other sets than balls. Consequently one has to apply different techniques in the general case. In [11] this was done in the special case when the set $P$ was open and it was proved that $\tilde{f}$ above exists if $P$ is (open and) connected. Of course this was not a generalization of the Rudin-Carleson theorem although it was enough to reprove the main result of [9]: Given any open connected set $P$ in a separable complex Banach space $X$ there exists a continuous function $\tilde{f}: \bar{A}-\{1\} \rightarrow X$, analytic on $\Delta$, whose range is contained and dense in $P$. 
In the present paper we study the general case when the set $P \subset X$ is not necessarily open. In the special case when $X=C$ we obtain a simple complete topological description of the sets $P \subset C$ having the following property: given any closed set $F \subset \partial \Delta$ of measure 0 and any $f \in C(F)$ satisfying $f(F) \subset P$ there exists an extension $\tilde{f} \in A(\Delta)$ of $f$ satisfying $\widetilde{f}(\bar{\Delta}) \subset P$. If $P \subset C$ has such a property and if $P$ consists of more than one point we show that for every relatively open set $B \subset \partial \Delta$, every relatively closed set $F \subset B$ of measure 0 and every continuous function $f: F \rightarrow P$ there exists a "peak" extension of $f$, i.e. an extension $\tilde{f} \in H_{B}(\Delta, C)$ of $f$ satisfying $\tilde{f}\left((\Delta \cup B)-F^{\prime}\right) \subset \operatorname{Int} P$. In the general case we study only the sets $P \subset X$ with the property that given any closed set $F \subset \partial \Delta$ of measure 0 , every function $f \in C(F, X)$ satisfying $f(F) \subset P$ admits a peak extension $\tilde{f} \in A(\Delta, X)$, and obtain their topological description.

In $\S 1$ we state the main results. In $\S 2$ we give the complete proofs; this section contains some lemmas and theorems which might be of independent interest. In $\S 3$ we present some simple applications to the ranges and cluster sets of analytic functions.

\section{Main results.}

Definition 1. $[10,11]$ Let $B \subset \partial \Delta$ be a relatively open set. $A$ subset $P$ of a complex Banach space $X$ is said to have the analytic extension property (AEP) with respect to $H_{B}(\Delta, X)$ if, given any relatively closed set $F \subset B$ of measure 0 and any continuous function $f: F \rightarrow P$ there exists an extension $\tilde{f} \in H_{B}(\Delta, X)$ of $f$ which satifies $\widetilde{f}(\Delta \cup B) \subset P$. We say that $P \subset X$ has AEP if it has AEP with respect to $H_{B}(\Delta, X)$ for every relatively open set $B \subset \partial \Delta$.

DEFINITION 2. Let $B \subset \partial \Delta$ be a relatively open set. A subset $P$ of a complex Banach space $X$ is said to have the peak analytic extension property (PAEP) with respect to $H_{B}(\Delta, X)$ if, given any $F$ and $f$ as above there exists an extension $\widetilde{f} \in H_{B}(\Delta, X)$ of $f$ satisfying $\widetilde{f}((\Delta \cup B)-F) \subset$ Int $P$. We call every such extension a peak extension with respect to $P$ (whether $P$ has $\mathrm{PAEP}$ or not). We say that $P \subset X$ has PAEP if it has PAEP with respect to $H_{B}(\Delta, X)$ for every relatively open set $B \subset \partial \Delta$.

Let $O$ be an open subset of a complex Banach space $X$. $O$ is called locally connected (LC) at a point $x \in X$ if given any $\varepsilon>0$ there exists $\delta>0$ such that if $\left(x+B_{\delta}(X)\right) \cap O$ is not empty it is contained in a connected component of $\left(x+B_{\varepsilon}(X)\right) \cap O$ [17]. Note that $O$ is LC at every point of $O$.

Now we are able to state our main results. 
THEOREM 1. Let $P$ be a nonempty subset of a complex Banach space $X$. Then the following are equivalent

(A) there exists a closed set $F \subset \partial \Delta$ of measure 0 with infinitely many points such that every continuous function $f: F \rightarrow P$ admits a peak extension $\tilde{f} \in A(\Delta, X)$ with respect to $P$

(B) $P$ has PAEP with respect to $A(\Delta, X)$

(C) $P$ has PAEP

(D) $P$ has the following properties:

(i) $P \subset \overline{\operatorname{Int} P}$.

(ii) Int $P$ is connected and locally connected at every point of $P$.

THEOREM 2. Let $P$ be a subset of $C$ containing more than one point. Then the following are equivalent

(A) there exists a closed set $F \subset \partial \Delta$ of measure 0 with infinitely many points such that every continuous function $f: F \rightarrow P$ admits an extension $\tilde{f} \in A(\Delta)$ satisfying $\widetilde{f}(\bar{\Delta}) \subset P$

(B) $P$ has AEP

(C) $P$ has PAEP.

Corollary. Let $P$ be a subset of $C$ containing more than one point. Then the following are equivalent

(A) given any closed set $F \subset \partial \Delta$ of measure 0 and any $f \in C\left(F^{\prime}\right)$ satisfying $f(F) \subset P$ there exists an extension $\tilde{f} \in A(\Delta)$ of $f$ satisfying $\widetilde{f}(\bar{\Delta}) \subset P$

(B) given any closed set $F \subset \partial \Delta$ of measure 0 and any $f \in C(F)$ satisfying $f(F) \subset P$ there exists an extension $\tilde{f} \in A(\Delta)$ of $f$ satisfying $\widetilde{f}(\bar{\Delta}-F) \subset \operatorname{Int} P$

(C) $P$ has the following properties

(i) $P \subset \overline{\operatorname{Int} P}$

(ii) Int $P$ is connected and locally connected at every point of $P$.

REMARK. The above Corollary gives a complete topological description of the sets $P \subset C$ for which the Rudin theorem holds. If $(C)$ in the Corollary above is satisfied by a (nonempty) compact set $P \subset C$ whose interior is simply connected note that then $P$ is homeomorphic to $\bar{d}[17]$. Finally, note that the main results of $[7,8,9$, 11] follow from Theorem 1 and Theorem 2 above.

\section{Proofs.}


LEMMA 1. Let $P$ be a nonempty subset of a complex Banach space $X$ and let $F \subset \partial \Delta$ be a closed set of measure 0 containing infinitely many points. Suppose that given any continuous function $f: F \rightarrow P$ there exists an extension $\tilde{f} \in A(\Delta, X)$ of $f$ such that $\widetilde{f}\left(\bar{J}-F^{\prime}\right) \subset \operatorname{Int} P . \quad$ Then

\section{(i) $P \subset \overline{\operatorname{Int} P}$}

(ii) Int $P$ is connected

(iii) Int $P$ is locally connected at every point of $P$.

Proof. Let $x \in P$. By the assumption there exists $\tilde{f} \in A(\Delta, X)$ satisfying $\tilde{f}(F)=\{x\}$ and $\tilde{f}(\bar{J}-F) \subset \operatorname{Int} P$. Let $\left\{z_{n}\right\} \subset \Delta$ converge to a point of $F$. By the continuity of $\tilde{f}\left\{\tilde{f}\left(z_{n}\right)\right\}$ converges to $x$. Since $\widetilde{f}\left(z_{n}\right) \in \operatorname{Int} P(n \in N)$ (i) is proved.

By the assumption $F$ is nowhere dense on $\partial \Delta$ and contains more than one point. Consequently $F=F_{1} \cup F_{2}$ where $F_{1}, F_{2}$ are nonempty disjoint compact sets. Let $x, y \in \operatorname{Int} P$ and define $f(s)=x\left(s \in F_{1}\right)$, $f(s)=y\left(s \in F_{2}\right)$. Clearly $f \in C(F, X)$ and by the assumption there exists $\tilde{f} \in A(\Delta, X)$ satisfying $\tilde{f}\left(F_{1}\right)=\{x\}, \quad \tilde{f}\left(F_{2}\right)=\{y\}$ and $\tilde{f}(\bar{\Delta}-F) \subset$ Int $P$. Let $z_{1} \in F_{1}, z_{2} \in F_{2}$. Now $t \mapsto \varphi(t)=\widetilde{f}\left(z_{1}+t\left(z_{2}-z_{1}\right)\right)$ is a path joining $x$ and $y$. By the properties of $f$ we have $\varphi(I) \subset \operatorname{Int} P$ which proves (ii).

To prove (iii) assume that Int $P$ is not $L C$ at a point $x \in P$. This means that there exists $\varepsilon>0$ such that for every $\delta>0$ the set $\left(x+B_{\delta}(X)\right) \cap \operatorname{Int} P$ meets at least two connected components of the set $\left(x+B_{\varepsilon}(X)\right) \cap$ Int $P$. It follows that there exist two sequences $\left\{x_{n}\right\} \subset \operatorname{Int} P,\left\{y_{n}\right\} \subset \operatorname{Int} P$ converging to $x$ such that for every $n \in N$ $x_{n}$ and $y_{n}$ lie in different components of $\left(x+B_{\varepsilon}(X)\right) \cap \operatorname{Int} P$. Assume for a moment that there exist a sequence $\left\{t_{n}\right\} \subset \partial \Delta$ converging to $t \in \partial \Delta$ and $\tilde{f} \in A(\Delta, X)$ satisfying, $\widetilde{f}(\Delta) \subset \operatorname{Int} P$ and $f\left(t_{2 n-1}\right)=x_{n}$, $f\left(t_{2 n}\right)=y_{n}(n \in N)$. By the continuity of $\widetilde{f}$ there exists a neighbourhood $U \subset \bar{J}$ of $t$ such that $\widetilde{f}(U) \subset x+B_{\varepsilon}(X)$. Consequently there is some $n \in N$ such that the closed segment $J$ joining $t_{2 n-1}$ and $t_{2 n}$ is contained in $U$. Then by the properties of $\tilde{f}, \widetilde{f}(J)$ is a path joining $x_{n}$ and $y_{n}$ in $\left(x+B_{s}(X)\right) \cap$ Int $P$, a contradiction.

It remains to prove the existence of $f$ and $\left\{t_{n}\right\}$ above. By the assumption $F$ contains infinitely many points which implies that there is some $t \in F$ which is a cluster point of $F-\{t\}$. Since $F$ is nowhere dense on $\partial \Delta$ there exists a decreasing sequence $\left\{T_{n}\right\}$ of open arcs in $\partial \Delta$ all of whose endpoints lie in $\partial \Delta-F$ and such that $F \subset T_{1}$, $\bigcap_{n=1}^{\infty} T_{n}=\{t\}$. Define $F_{n}=F \cap\left(T_{n}-T_{n+1}\right)(n \in N)$. Then $F_{n}(n \in N)$ are disjoint compact sets infinitely many of which are not empty. Passing to a subsequence if necessary we may assume that all sets $F_{n}(n \in N)$ are nonempty. Define $f: F \rightarrow X$ by 


$$
\begin{aligned}
& f(s)=x_{n}\left(s \in F_{2 n-1} ; n \in N\right), \\
& f(s)=y_{n}\left(s \in F_{2 n} ; n \in N\right), \\
& f(t)=x .
\end{aligned}
$$

It is easy to see that $f$ is continuous. By the assumption there exists an extension $\tilde{f} \in A(\Delta, X)$ of $f$ satisfying $\tilde{f}\left(\bar{\Delta}-F^{\prime}\right) \subset \operatorname{Int} P$. Choose $t_{n} \in F_{n}(n \in N)$.

An open subset $O$ of a complex Banach space $X$ is called uniformly locally connected (ULC) on a subset $K$ of $X$ if given any $\varepsilon>0$ there exists $\delta>0$ such that for every $x \in K$ the set $\left(x+B_{\delta}(X)\right) \cap O$, if not empty, is contained in a connected component of the set $\left(x+B_{s}(X)\right) \cap O[17]$. We call any such $\varepsilon \mapsto \delta(\varepsilon)$ a modulus of ULC of $O$ on $K$.

Lemma 2. Let $O$ be an open subset of a complex Banach space $X$ and let $K \subset X$ be a compact set. Suppose that $O$ is locally connected at each point $x \in K$. Then $O$ is uniformly locally connected on $K$.

Proof. Simple. For the idea see [17, p. 160].

LEMma 3. [11] Let $B \subset \partial \Delta$ be a relatively open set, $G \subset B$ a relatively closed set of measure 0 , and $H \subset B$ a compact set of measure 0, disjoint from $G$. Let $U \subset \Delta \cup B$ be a neighbourhood of $H$ and let $\varepsilon>0$. Assume that $P$ is an open connected set in a complex Banach space $X$ which contains the point 0 and let $f: H \rightarrow P$ be a continuous function. There exists $\tilde{f} \in H_{B}(\Delta, X)$ which satisfies

(i ) $\widetilde{f} \mid H=f$

(ii) $\tilde{f}(G)=\{0\}$

(iii) $\|\widetilde{f}(z)\|<\varepsilon(z \in(\Delta \cup B)-U)$

(iv) $\tilde{f}(\Delta \cup B) \subset P$.

Lemma 3, which gives an approximate solution to our Problem, is the most important tool in the present paper.

Lemma 4. Let $O$ be an open connected set in a complex Banach space $X$. Let $F \subset \partial \Delta$ be a closed set of measure 0 and $f_{1}, f_{2}$ two continuous functions from $F$ to $O$. There exists a homotopy $p: F \times I \rightarrow O$ satisfying

$$
\left.\begin{array}{l}
p(s, 0)=f_{1}(s) \\
p(s, 1)=f_{2}(s)
\end{array}\right\}(s \in F) .
$$


Proof. Choose $s_{0} \in \partial \Delta-F$ and $x \in O$. By Lemma 3 there exist $g_{i} \in A(\Delta, X)(i=1,2)$ satisfing

$$
\left.\begin{array}{l}
g_{i} \mid F=f_{i} \\
g_{2}(\bar{\Delta}) \subset O \\
g_{i}\left(s_{0}\right)=x
\end{array}\right\}(i=1,2) .
$$

Put

$$
p(s, t)= \begin{cases}g_{1}\left(s+2 t\left(s_{0}-s\right)\right) & (s \in F, 0 \leqq t \leqq 1 / 2) \\ g_{2}\left(s_{0}-(1-2 t)\left(s-s_{0}\right)\right) & (s \in F, 1 / 2 \leqq t \leqq 1)\end{cases}
$$

It is easy to check that $p$ has the required properties.

Lemma 5. Let $O$ be an open subset of a complex Banach space and let $F \subset \partial \triangle$ be a closed set of measure 0. Suppose that $f: F \rightarrow \bar{O}$ is a continuous function and assume that $O$ is ULC on $f(F)$. Let $\varepsilon \mapsto \delta(\varepsilon)$ be a modulus of ULC of $O$ on $f(F)$. Let $R>0$ and assume that $g: F \rightarrow O$ is a continuous function satisfying

$$
\|f(s)-g(s)\|<\delta(R) / 2 \quad(s \in F) .
$$

Let $\varepsilon>0$. There exists a homotopy $\pi: F \times I \rightarrow O$ satisfying

(i) $\pi(s, 0)=g(s) \quad(s \in F)$,

(ii) $\|\pi(s, 1)-f(s)\|<\varepsilon \quad(s \in F)$,

(iii) $\|\pi(s, t)-f(s)\|<2 R \quad(s \in F, 0 \leqq t \leqq 1)$.

Proof. By the properties of $F$

$$
F=\bigcup_{i=1}^{m} F_{\imath}
$$

where $F_{\imath}(i=1,2, \cdots, m)$ are disjoint compact sets such that

$$
\|f(\xi)-f(\eta)\|<\min \{R, \delta(R) / 2, \varepsilon / 2\} \quad\left(\xi, \eta \in F_{i} ; i=1,2, \cdots, m\right) .
$$

Choose $\zeta_{i} \in F_{i}(i=1,2, \cdots, m)$. Since $f(F) \subset \bar{O}$ there exist $x_{\imath} \in O$ $(i=1,2, \cdots, m)$ such that

$$
\left\|f\left(\zeta_{i}\right)-x_{i}\right\|<\min \{\delta(R) / 2, \varepsilon / 2\} \quad(i=1,2, \cdots, m) .
$$

Define $h(s)=x_{i}\left(s \in F_{i} ; i=1,2, \cdots, m\right)$. By the assumption and by (1.1) we have

$$
\begin{aligned}
\left\|f\left(\zeta_{i}\right)-g(s)\right\| & \leqq\left\|f\left(\zeta_{i}\right)-f(s)\right\|+\|f(s)-g(s)\| \\
& <\delta(R) \quad\left(s \in F_{i} ; i=1,2, \cdots, m\right)
\end{aligned}
$$

and similarly by (1.1) and (1.2) 


$$
\begin{aligned}
\|h(s)-f(s)\| & \leqq\left\|h(s)-f\left(\zeta_{i}\right)\right\|+\left\|f\left(\zeta_{i}\right)-f(s)\right\| \\
& <\varepsilon \quad\left(s \in F_{i} ; i=1,2, \cdots, m\right) .
\end{aligned}
$$

By (1.2) and (1.3) we have

$$
x_{i}=h(s) \in\left(f\left(\zeta_{i}\right)+B_{\hat{o}(R)}(X)\right) \cap O \quad\left(s \in F_{i} ; i=1,2, \cdots, m\right)
$$

and

$$
g(s) \in\left(f\left(\zeta_{i}\right)+B_{\hat{o}(R)}(X)\right) \cap O \quad\left(s \in F_{i} ; i=1,2, \cdots, m\right)
$$

and it follows that for each $i=1,2, \cdots, m$ both $h\left(F_{i}\right)$ and $g\left(F_{i}\right)$ are contained in the same connected component of $\left(f\left(\zeta_{i}\right)+B_{R}(X)\right) \cap O$. Now Lemma 4 applies to show that for each $i=1,2, \cdots, m$ there exists a homotopy $p_{i}: F_{i} \times I \rightarrow O$ satisfying

$$
\left.\begin{array}{l}
p_{i}(s, 0)=g(s) \\
p_{i}(s, 1)=h(s)
\end{array}\right\}\left(s \in F_{i} ; i=1,2, \cdots, m\right)
$$

and

$$
\begin{aligned}
& p_{i}(s, t) \in\left(f\left(\zeta_{i}\right)\right.\left.+B_{R}(X)\right) \cap O \\
&\left(s \in F_{i} ; 0 \leqq t \leqq 1 ; i=1,2, \cdots, m\right) .
\end{aligned}
$$

Define $\pi: F \times I \rightarrow O$ by $\pi \mid F_{i} \times I=p_{i}(i=1,2, \cdots, m)$. Since $F_{i}$ $(i=1,2, \cdots, m)$ are disjoint compact sets it follows that $F_{i} \times I$ $(i=1,2, \cdots, m)$ are disjoint compact subsets of $F \times I$ and consequently $\pi$ is continuous. Clearly $\pi(s, 0)=g(s)(s \in F)$. By (1.1) and (1.5) we have

$$
\begin{aligned}
\left\|p_{i}(s, t)-f(s)\right\| & \leqq\left\|p_{2}(s, t)-f\left(\zeta_{i}\right)\right\|+\left\|f\left(\zeta_{i}\right)-f(s)\right\| \\
& <2 R \quad\left(s \in F_{i} ; 0 \leqq t \leqq 1 ; i=1,2, \cdots, m\right)
\end{aligned}
$$

which implies (iii). Finally, by (1.4) we have $\|\pi(s, 1)-f(s)\|<\varepsilon$ $(s \in F)$.

THEOREM 3. Let $O$ be an open subset of a complex Banach space $X$ and let $F \subset \partial \Delta$ be a closed set of measure 0 . Let $f: F \rightarrow \bar{O}$ be a continuous function and assume that $O$ is locally connected at each point of $f(F)$. Then there exists a homotopy $p: F \times I \rightarrow \bar{O}$ satisfying

(i) $p(s, t) \in O(s \in F ; 0 \leqq t<1)$

(ii) $p(s, 1)=f(s)(s \in F)$.

Proof. Observe first that $f(F)$ being compact $O$ is ULC on $f(F)$ by Lemma 2. Let $\varepsilon \mapsto \delta(\varepsilon)$ be a modulus of ULC of $O$ on $f(F$.) Choose a decreasing sequence $\left\{R_{n}\right\}$ of positive numbers converging to 0 and a strictly increasing sequence $\left\{t_{n}\right\}$ of nonnegative numbers converging to 1 where $t_{1}=0$. 
Assume for a moment that there exists a continuous function $p: F \times(I-\{1\}) \rightarrow O$ satisfying

$$
\|p(s, t)-f(s)\|<2 R_{n} \quad\left(s \in F ; t_{n} \leqq t<1 ; n \in N\right) .
$$

Since $\left\{R_{n}\right\}$ converges to 0 we have $\lim _{t \rightarrow 1} p(s, t)=f(s)$ uniformly for $s \in F$. Since $f$ is continuous on $F$ it follows that the extension $p: F \times I \rightarrow \bar{O}$ of $p$ defined on $F \times\{1\}$ by $p(s, 1)=f(s)(s \in F)$ is continuous on $F \times I$ and satisfies (i), (ii) above.

To prove the existence of $p$ satisfying (2.1) we first prove that there exists a sequence of homotopies $\pi_{n}: F \times I \rightarrow O(n \in N)$ satisfying

$$
\begin{gathered}
\pi_{n+1}(s, 0)=\pi_{n}(s, 1) \quad(s \in F, n \in N) \\
\left\|\pi_{n}(s, 1)-f(s)\right\|<\delta\left(R_{n+1}\right) / 2 \quad(s \in F, n \in N)
\end{gathered}
$$

and

$$
\left\|\pi_{n}(s, t)-f(s)\right\|<2 R_{n} \quad(s \in F, 0 \leqq t \leqq 1, n \in N) .
$$

To define $\pi_{1}$ observe that $F=\bigcup_{i=1}^{m} F_{i}$ where $F_{i}(i=1,2, \cdots, m)$ are disjoint compact sets such that

$$
\|f(\xi)-f(\eta)\|<\delta\left(R_{1}\right) / 4 \quad\left(\xi, \eta \in F_{. i}, i=1,2, \cdots, m\right)
$$

Choose $\zeta_{i} \in F_{i}(i=1,2, \cdots, m)$. Since $f(F) \subset \bar{O}$ there exist $x_{i} \in \bar{O}$ $(i=1,2, \cdots, m)$ satisfying

$$
\left\|x_{i}-f\left(\zeta_{i}\right)\right\|<\delta\left(R_{1}\right) / 4 \quad(i=1,2, \cdots, m) .
$$

Define $g(s)=x_{i}\left(s \in F_{i}, i=1,2, \cdots, m\right)$. Then $g: F \rightarrow O$ is a continuous function which satisfies $\|f(s)-g(s)\|<\delta\left(R_{1}\right) / 2 \quad(s \in F)$ by (2.5) and (2.6). By Lemma 5 applied to $f, g, R=R_{1}$ and $\varepsilon=\delta\left(R_{2}\right) / 2$ there exists a homotopy $\pi_{1}: F \times I \rightarrow O$ satisfying $\left\|\pi_{1}(s, 1)-f(s)\right\|<\delta\left(R_{2}\right) / 2$ $(s \in F)$ and $\left\|\pi_{1}(s, t)-f(s)\right\|<2 R_{1}(s \in F, 0 \leqq t \leqq 1)$.

Assume that there exist $\pi_{n}(n=1,2, \cdots, l)$ satisfying (2.2) for $1 \leqq n \leqq l-1$ and (2.3), (2.4) for $1 \leqq n \leqq l$. By Lemma 5 applied to $f$, to $s \mapsto g(s)=\pi_{l}(s, 1)(s \in F)$, to $R=R_{l+1}$ and $\varepsilon=\delta\left(R_{l+2}\right) / 2$ there exists a homotopy $\pi_{l+1}: F \times I \rightarrow O$ satisfying (2.2) for $n=l$ and (2.3), (2.4) for $n=l+1$. Now the existence of $\left\{\pi_{n}: n \in N\right\}$ satisfying (2.2), (2.3) and (2.4) follows by induction.

Now define $p\left(s, t_{n}+t\left(t_{n+1}-t_{n}\right)\right)=\pi_{n}(s, t)(s \in F, 0 \leqq t \leqq 1, n \in N)$. It is easy to check that $p$ has all the required properties.

Lemma 6. Let $O$ be an open subset of a complex Banach space $X$. Let $B \subset \partial \Delta$ be a relatively open set, $H \subset B$ a compact set of measure 0 and $G \subset B$ a relatively closed set of measure 0 , disjoint from $H$. Suppose that $\pi: H \times I \rightarrow O$ is a continuous function 
satisfying

$$
\operatorname{diam}\{\pi(s, t): 0 \leqq t \leqq 1\} \leqq M \quad(s \in H)
$$

for some $M$. Let $U \subset \Delta \cup B$ be a neighbourhood of $H$ and let $\varepsilon>0$ be arbitrary. Assume that $h: \Delta \cup B \rightarrow O$ is a continuous function such that

(a) $h(s)=\pi(s, 0)(s \in H)$

(b) there exists $\tau>0$ such that

$$
h(\Delta \cup B)+B_{i}(X) \subset O .
$$

Then there exists $g \in H_{B}(\Delta, X)$ satisfying

(i) $(h+g)(s)=\pi(s, 1) \quad(s \in H)$

(ii) $g(s)=0 \quad(s \in G)$

(iii) $\|g(z)\|<\varepsilon \quad(z \in(\Delta \cup B)-U)$

(iv) $\|g(z)\|<M+\varepsilon \quad(z \in \Delta \cup B)$

(v) there exists $\delta>0$ such that

$$
(h+g)(\Delta \cup B)+B_{\delta}(X) \subset O \text {. }
$$

Proof. By the assumption $\pi: H \times I \rightarrow O$ is continuous hence $\pi(H \times I)$ is a compact set contained in $O$. Consequently by (b) there exists $\delta>0$ which satisfies

$$
\begin{gathered}
\delta<\varepsilon / 3 \\
h(\Delta \cup B)+B_{2 \delta}(X) \subset O
\end{gathered}
$$

and

$$
\pi(H \times I)+B_{5 \delta}(X) \subset O .
$$

By the properties of $H$

$$
H=\bigcup_{j=1}^{m} H_{j}
$$

where $H_{j}(j=1,2, \cdots, m)$ are disjoint compact sets such that

$$
\begin{aligned}
& \|\pi(\xi, 0)-\pi(\eta, 0)\|<\delta \\
& \|\pi(\xi, 1)-\pi(\eta, 1)\|<\delta \quad\left(\xi, \eta \in F_{j}: j=1,2, \cdots, m\right) .
\end{aligned}
$$

Since $h$ is continuous on $\Delta \cup B$ there exist disjoint neighbourhoods $V_{j} \subset U$ of the sets $H_{j}(j=1,2, \cdots, m)$, respectively, such that

$$
\|h(\xi)-h(\eta)\|<\delta \quad\left(\xi, \eta \in V_{j}: j=1,2, \cdots, m\right) .
$$

Let $1 \leqq j \leqq m$. Choose $\xi_{j} \in H_{j}$ and consider the set

$$
P_{j}=-\pi\left(\xi_{j}, 0\right)+\left\{\pi\left(\xi_{j}, t\right): 0 \leqq t \leqq 1\right\}+B_{2 \delta}(X) .
$$


By the continuity of $\pi, p_{j}$ is an open connected set in $X$ containing the point 0. By (3.3) we have

$$
\left.\begin{array}{l}
\pi(s, 0) \in \pi\left(\xi_{j}, 0\right)+B_{\delta}(X) \\
\pi(s, 1) \in \pi\left(\xi_{j}, 1\right)+B_{\delta}(X)
\end{array}\right\}\left(s \in H_{j}\right)
$$

and it follows that $\pi(s, 1)-\pi(s, 0) \in P_{j}\left(s \in H_{j}\right)$. Hence $s \mapsto \pi(s, 1)-$ $\pi(s, 0)$ is a continuous function from $H_{j}$ into $P_{j}$. By Lemma 3 there exists $g_{j} \in H_{B}(\Delta, X)$ satisfying

$$
\begin{gathered}
g_{j}(s)=\pi(s, 1)-\pi(s, 0) \quad\left(s \in H_{j}\right) \\
g_{j}(s)=0 \quad\left(s \in G \cup\left(H-H_{j}\right)\right) \\
\left\|g_{j}(z)\right\|<\delta / m \quad\left(z \in(\Delta \cup B)-V_{j}\right) \\
g_{j}(\Delta \cup B) \subset P_{j} .
\end{gathered}
$$

Define

$$
g=\sum_{j=1}^{m} g_{j}
$$

By (3.7) we have

$$
\|g(z)\|<\delta \quad\left(z \in(\Delta \cup B)-\bigcup_{j=1}^{m} V_{j}\right) .
$$

Since $\quad V_{j} \subset U \quad(j=1,2, \cdots, m) \quad$ it follows that $\|g(z)\|<\delta$ $(z \in(\Delta \cup B)-U)$ and by (3.0) (iii) is satisfied.

If $z \in V_{j}$ for some $j, 1 \leqq j \leqq m$ then $z \notin V_{k}(k \neq j, 1 \leqq k \leqq m)$. Consequently we have by (3.7) and (3.8)

$$
\begin{aligned}
g(z)= & g_{j}(z)+\sum_{k \neq j} g_{k}(z) \\
& \in P_{j}+B_{\delta}(X) \quad\left(z \in V_{j}, j=1,2, \cdots, m\right)
\end{aligned}
$$

By the assumption $\operatorname{diam}\{\pi(s, t): 0 \leqq t \leqq 1\} \leqq M(s \in H)$ which implies that

$$
\sup _{y \in P_{j}}\|y\| \leqq M+2 \delta \quad(j=1,2, \cdots, m) .
$$

By (3.0), (3.9) and (3.10) (iv) follows.

Now, (3.9) implies that

$$
h(z)+g(z) \in h(\Delta \cup B)+B_{\delta}(X) \quad\left(z \in(\Delta \cup B)-\bigcup_{j=1}^{m} V_{j}\right)
$$

and by (3.1) it follows that

$$
h(z)+g(z)+B_{\delta}(X) \subset O \quad\left(z \in(\Delta \cup B)-\bigcup_{j=1}^{m} V_{j}\right) .
$$


If $z \in V_{j}$ for some $j, 1 \leqq j \leqq m$ then $z \notin V_{k}(k \neq j, 1 \leqq k \leqq m)$ so by (a), by (3.4) and by (3.10) it follows that

$$
\begin{aligned}
h(z) & +g(z) \in h\left(\xi_{j}\right)+B_{\hat{o}}(X)+P_{j}+B_{\hat{o}}(X) \\
& =h\left(\xi_{j}\right)+B_{\hat{o}}(X)-\pi\left(\xi_{j}, 0\right)+\left\{\pi\left(\xi_{j}, t\right): 0 \leqq t \leqq 1\right\}+B_{2 \hat{\delta}}(X)+B_{\hat{o}}(X) \\
& \subset \pi(F \times I)+B_{4 \hat{\delta}}(X) .
\end{aligned}
$$

Now (3.2) implies that $h(z)+g(z)+B_{\hat{o}}(X) \subset O\left(z \in \bigcup_{j=1}^{m} V_{j}\right)$, which, together with (3.11) gives (v).

By the properties of $g_{j}(1 \leqq j \leqq m)$ it is easy to see that (i) and (ii) are also satisfied.

THEOREM 4. Let $O$ be an open connected subset of a complex Banach space $X$. Let $B \subset \partial \Delta$ be a relatively open set and $F \subset B a$ relatively closed set of measure 0 . Assume that $p: F \times I \rightarrow \bar{O}$ is a continuous function satisfying $p(s, t) \in O(s \in F, 0 \leqq t<1)$.

There exists $f \in H_{B}(\Delta, X)$ such that

(a) $f(s)=p(s, 1) \quad(s \in F)$

(b) $f(z) \in O \quad(z \in(\Delta \cup B)-F)$.

REMARK. In particular, Theorem 4 implies the following: Let $O, B$ and $F$ be as above and let $u: F \rightarrow \bar{O}$ be a continuous function. If there exists a peak continuous extension of $u$, i.e., a continuous extension $v: \Delta \cup B \rightarrow O$ of $u$ satisfying $v((\Delta \cup B)-F) \subset O$ then there exists a peak analytic extension of $u$, i.e. a continuous extension $w: \Delta \cup B \rightarrow \bar{O}$ of $u$, analytic on $\Delta$ and satisfying $w((\Delta \cup B)-F) \subset O$. To see this, put $p(s, t)=v(s t)(s \in F, 0 \leqq t \leqq 1)$ and apply Theorem 4 .

Proof of Theorem 4. We consider only the case when $F$ is not compact. It is easy to see how to simplify the proof below in the case of compact $F$; note that in the latter case the proof is considerably simpler.

Part 1. With no loss of generality we may assume that $0 \in O$. As in [8] write

$$
F=\bigcup_{j=1}^{\infty} F_{j}
$$

where $F_{j} \subset \Delta \cup B(j \in N)$ are compact sets infinitely many of which are not empty, such that there exist disjoint open sets $0_{j} \subset \Delta \cup B$ $(j \in N)$ with the property that $F_{j} \subset 0_{j}(j \in N)$ and such that every compact subset of $\Delta \cup B$ misses all but a finite number of the sets $0_{j}$. Passing to a subsequence if necessary we may assume that all 
sets $F_{j}(j \in N)$ are nonempty. Passing to a smaller $0_{1}$ if necessary we may assume that $0_{1}$ is contained in a compact subset of $\Delta \cup B$.

By Lemma 4 there exists for each $j \in N$ a homotopy $p_{j}: F \times I \rightarrow O$ satisfying $p_{j}(s, 0)=0, \quad p_{j}(s, 1)=p(s, 0) \quad\left(s \in F_{j}\right)$. With no loss of generality we may assume that $p(s, 0)=0 \quad(s \in F)$ (otherwise we replace $p$ by $\pi$ defined as follows

$$
\begin{aligned}
& \pi(s, t)=p_{j}(s, 2 t) \\
& \left(0 \leqq t \leqq 1 / 2, s \in F_{j}, j \in N\right) \\
& \pi(s, t)=p(s, 2 t-1) \\
& (1 / 2 \leqq t \leqq 1, s \in F) \text {; }
\end{aligned}
$$

by the properties of the sets $F_{j}(j \in N)$ it is easy to see that $\pi: F \times I \rightarrow \bar{O}$ is continuous).

By the compactness of the sets $F_{j}(j \in N)$ and by the continuity of $p$ there exists for each $j \in N$ an increasing sequence $\left\{t_{i j}: i \in N\right\}$, $0<t_{i j}<1(i \in N)$, converging to 1 and satisfying

$$
\operatorname{diam}\left\{p(s, t): t_{i j} \leqq t \leqq 1\right\}<1 / 2^{i+2} \quad\left(s \in F_{j}, j \in N, i \in N\right) .
$$

Part 2. In the sequel we will prove the following:

(A) for each $j \in N$ there exists a decreasing sequence $U_{i j} \subset \Delta \cup B$ $(i \in N)$ of neighbourhoods of $F_{j}$ contained in $0_{j}$ and satisfying

$$
\bigcap_{i=1}^{\infty} U_{i j}=F_{j} \quad(j \in N)
$$

(B) there exists a decreasing sequence $\left\{\varepsilon_{i}\right\}$ of positive numbers where

$$
B_{\varepsilon_{1}}(X) \subset O
$$

such that

(C) there exists a sequence $\left\{g_{i}\right\} \subset H_{B}(\Delta, X)$ satisfying

(i ) $\left(\sum_{m=1}^{i} g_{m}\right)(s)=p\left(s, t_{i j}\right) \quad\left(s \in F_{j}, 1 \leqq j \leqq i, i \in N\right)$

$$
g_{i}(s)=0 \quad\left(s \in F_{j}, j>i, j \in N, i \in N\right)
$$

(ii) $\left\|g_{i}(z)\right\|<1 / 2^{i} \quad\left(z \in(\Delta \cup B)-U_{i i}, i \in N\right)$

(iii) $\left\|g_{i}(z)\right\|<\varepsilon_{i} / 2^{i} \quad\left(z \in(\Delta \cup B)-\bigcup_{j=1}^{i} U_{i j}, i \in N\right)$

(iv) $\left(\sum_{m=1}^{i} g_{m}\right)(\Delta \cup B)+\varepsilon_{i+1}(X) \subset O \quad(i \in N)$.

Assume for a moment that we have proved (A), (B), (C) above. Define

$$
f(z)=\sum_{i=1}^{\infty} g_{i}(z) \quad(z \in \Delta \cup B) .
$$

We show that $f$ has all the required properties.

Since each compact of $\Delta \cup B$ misses all but a finite number of the sets $0_{j},(\mathrm{C})$ (ii) implies that the series converges uniformly on 
each compact subset of $\Delta \cup B$. Consequently $f \in H_{B}(\Delta, X)$. By (C) (i) and by the continuity of $p$ we have for all $j \in N$ and for all $s \in F_{j}$

$$
f(s)=\lim _{n \rightarrow \infty} \sum_{i=1}^{n} g_{i}(s)=\lim _{\substack{n \rightarrow \infty \\ n>j}} \sum_{i=1}^{n} g_{i}(s)=\lim _{n \rightarrow \infty} p\left(s, t_{n j}\right)=p(s, 1) .
$$

Consequently $f(s)=p(s, 1)(s \in F)$ so (a) is satisfied.

To check (b), let $z \in \Delta \cup B-F$. Let first $z \in(\Delta \cup B)-\bigcup_{j=1}^{\infty} U_{1 j}$. Now (C) (iii) implies that $\|f(z)\|<\varepsilon_{1}$ and by (4.2) it follows that $f(z) \in O$. Now, let $z \in U_{1 j}$ for some $j \in N$. Then $z \notin U_{1 k}(k \neq j, k \in N)$. Further, since $z \notin F_{j}$ it follows that there exists $i \in N$ such that $z \in U_{i j}, \quad z \notin U_{i+1, j}$. Consequently $z \in(\Delta \cup B)-\bigcup_{j=1}^{\infty} U_{i+1, j}$, so that $z \in(\Delta \cup B)-\bigcup_{j=1}^{\infty} U_{k j}$ for every $k \in N, k \geqq i+1$. By (C) (iii) it follows that

$$
\left\|\sum_{k=i+1}^{\infty} g_{k}(z)\right\|<\varepsilon_{i+1}
$$

which, by (C) (iv) implies that $f(z) \in O$.

Part 3. It remains to prove (A), (B), (C) above and we do this by induction.

First, choose $\varepsilon_{1}>0$ such that (4.2) holds and put $U_{11}=0_{1}$. Choose a decreasing sequence $\left\{U_{i 1}\right\}$ of neighbourhoods of $F_{1}$ in $\triangle \cup B$ contained in $0_{1}$ and satisfying $\bigcap_{i=1}^{\infty} U_{i 1}=F_{1}$. By Lemma 3 there exists $g_{1} \in H_{B}(\Delta, X) \quad$ satisfying $g_{1}(s)=p\left(s, t_{11}\right) \quad\left(s \in F_{1}\right), \quad g_{1}(s)=0$ $\left(s \in F-F_{1}\right), g_{1}(\Delta \cup B) \subset O$ and

$$
\left\|g_{1}(z)\right\|<\min \left\{1 / 2, \varepsilon_{1} / 2\right\} \quad\left(z \in(\Delta \cup B)-U_{11}\right) .
$$

Now $0_{1}$ is contained in a compact subset of $\triangle \cup B$. Consequently $g_{1}\left(U_{11}\right)$ is contained in a compact set contained in $O$ by the continuity of $g_{1}$. It follows by (4.2) and (4.3) that there exists $\varepsilon_{2}, 0<\varepsilon_{2}<\varepsilon_{1}$ satisfying $g_{1}(\Delta \cup B)+B_{\varepsilon_{2}}(X) \subset O$. So we have proved the existence of a sequence $\left\{U_{k 1}, k \in N\right\} \varepsilon_{1}, \varepsilon_{2}<\varepsilon_{1}$ and $g_{1} \in H_{B}(\Delta, X)$ satisfying (A) and (C) (i)-(iv) for $i=1$.

Assume that we have proved the existence of the sequences $\left\{U_{k j}, k \in N\right\}(1 \leqq j \leqq n)$, of a decreasing sequence $\left\{\varepsilon_{k}, 1 \leqq k \leqq n+1\right\}$ of positive numbers and of a sequence $\left\{g_{i}, 1 \leqq i \leqq n\right\} \subset H_{B}(\Delta, X)$ such that (A) is satisfied for $1 \leqq j \leqq n$ and (C) (i)-(iv) is satisfied for $1 \leqq i \leqq n$. We show below how to choose a sequence $\left\{U_{k, n+1}, k \in N\right\}$ to satisfy (A) for $j=n+1$ and then how to choose $\varepsilon_{n+2}$ : $0<\varepsilon_{n+2}<$ $\varepsilon_{n+1}$ and $g_{n+1} \in H_{B}(\Delta, X)$ to satisfy (C) (i)-(iv) for $i=n+1$.

By the compactness of the set $p\left(F_{n+1} \times\left[0, t_{n+1, n+1}\right]\right)$ there exist $\delta>0$ and $\varepsilon>0$ satisfying

$$
p\left(F_{n+1} \times\left[0, t_{n+1, n+1}\right]\right)+B_{4 \delta}(X) \subset O
$$




$$
\begin{gathered}
4 \delta<\varepsilon_{n+1} \\
\delta+\varepsilon<1 / 2^{n+1}-1 / 2^{n+2} \\
\delta+\varepsilon<\varepsilon_{n+1} / 2^{n+1} .
\end{gathered}
$$

We choose $\left\{U_{k, n+1}, k \in N\right\}$ as follows: $\sum_{m=1}^{n} g_{m}$ is continuous on $\Delta \cup B$ and by (C) (i) $\left(\sum_{m=1}^{n} g_{m}\right)(s)=0\left(s \in F_{n+1}\right)$. Consequently there exists a neighbourhood $U_{n+1, n+1} \subset 0_{n+1}$ of $F_{n+1}$ in $\Delta \cup B$ such that

$$
\left\|\left(\sum_{m=1}^{n} g_{m}\right)(z)\right\|<\delta \quad\left(z \in U_{n+1, n+1}\right) \text {. }
$$

Now choose a decreasing sequence $U_{k, n+1} \subset 0_{n+1}(k \in N)$ of neighbourhoods of $F_{n+1}$ in $\Delta \cup B$ satisfying $U_{k, n+1}=U_{n+1, n+1}(k \in N, k \leqq n)$ and $\bigcap_{k=1}^{\infty} U_{k, n+1}=F_{n+1}$, so that (A) is satisfied for $j=n+1$.

By Lemma 3 there exists $e \in H_{B}(\Delta, X)$ satisfying

$$
\begin{gathered}
e(s)=p\left(s, t_{n+1, n+1}\right) \quad\left(s \in F_{n+1}\right) \\
e(s)=0 \quad\left(s \in F_{k}, k \in N, k \neq n+1\right) \\
\|e(z)\|<\delta \quad\left(z \in(\Delta \cup B)-U_{n+1, n+1}\right)
\end{gathered}
$$

and

$$
e(\Delta \cup B) \subset p\left(F_{n+1} \times\left[0, t_{n+1, n+1}\right]\right)+B_{\delta}(X)
$$

since $p\left(F_{n+1} \times\left[0, t_{n+1, n+1}\right]\right)+B_{\delta}(X)$ is an open connected set which contains 0 and the set $\left\{p\left(s, t_{n+1, n+1}\right), s \in F_{n+1}\right\}$.

Consider the function $h=\sum_{m=1}^{n} g_{m}+e$. Since (C) (iv) holds for $i=n$ it follows by (4.5) that $\left(\sum_{m=1}^{n} g_{m}\right)(\Delta \cup B)+B_{4 \delta}(X) \subset O$ which, by (4.11) implies that

$$
h(z)+B_{2 \delta}(X) \subset O \quad\left(z \in(\Delta \cup B)-U_{n+1, n+1}\right) .
$$

Now let $z \in U_{n+1, n+1}$. By (4.8) and (4.12) we have $h(z) \in p\left(F_{n+1} \times\right.$ $\left.\left[0, t_{n+1, n+1}\right]\right)+B_{2 \delta}(X)$ which, by (4.4) implies that $h(z)+B_{2 \delta}(X) \subset O$ $\left(z \in U_{n+1, n+1}\right)$ and by (4.13) it follows that

$$
h(\Delta \cup B)+B_{2 \delta}(X) \subset O \text {. }
$$

Now, put

$$
\begin{aligned}
H & =\bigcup_{k=1}^{n+1} F_{k}, \quad G=F-H, \\
\pi(s, t) & = \begin{cases}p\left(s, t_{n j}+t\left(t_{n+1, j}-t_{n j}\right)\right. & \left(s \in F_{j}, 1 \leqq j \leqq n\right) \\
p\left(s, t_{n+1, n+1}\right) & \left(s \in F_{n+1}\right),\end{cases} \\
U & =\bigcup_{k=1}^{n+1} U_{n+1, k}, \quad M=1 / 2^{n+2} .
\end{aligned}
$$


Observe that by (C) (i), (4.9) and (4.10) $\pi(s, 0)=h(s)(s \in H)$. Note also that by (4.1) diam $\{\pi(s, t), 0 \leqq t \leqq 1\}<M(s \in H)$. Now Lemma 6 applies to show that there exists $g \in H_{B}(\Delta, X)$ with the following properties:

$$
\begin{gathered}
(h+g)(s)=\pi(s, 1)=p\left(s, t_{n+1, j}\right) \quad\left(s \in F_{j}, 1 \leqq j \leqq n+1\right) \\
g(s)=0 \quad\left(s \in F_{j}, j \in N, j \geqq n+2\right) \\
\|g(z)\|<\varepsilon \quad\left(z \in(\Delta \cup B)-\bigcup_{k=1}^{n+1} U_{n+1, k}\right) \\
\|g(z)\|<1 / 2^{n+2}+\varepsilon \quad(z \in \Delta \cup B),
\end{gathered}
$$

and that there exists $\varepsilon_{n+2}, 0<\varepsilon_{n+2}<\varepsilon_{n+1}$ such that

$$
(h+g)(\Delta \cup B)+B_{\varepsilon_{n+2}}(X) \subset 0 .
$$

Define $g_{n+1}=e+g$. Clearly $g_{n+1} \in H_{B}(\Delta, X)$. By (4.15) and (4.16) it follows that (C) (i) is satisfied for $i=n+1$. Further, by (4.19) (C) (iv) is satisfied for $i=n+1$.

Let $z \in(\Delta \cup B)-U_{n+1, n+1}$. By (4.11), (4.18) and (4.6) we have $\left\|g_{n+1}(z)\right\| \leqq\|e(z)\|+\|g(z)\|<\delta+\varepsilon+1 / 2^{n+2}<1 / 2^{n+1}$ which implies that (C) (ii) is satisfied for $i=n+1$. Finally, let $z \in(\Delta \cup B)-$ $\bigcup_{j=1}^{n+1} U_{n+1, j}$. By (4.11), (4.17) and (4.7) it follows that

$$
\left\|g_{n+1}(z)\right\|<\delta+\varepsilon<\varepsilon_{n+1} / 2^{n+1}
$$

which implies that (C) (iii) is also satisfied for $i=n+1$.

Proof of Theorem 1. Clearly $(\mathrm{C}) \Rightarrow(\mathrm{B}) \Rightarrow(\mathrm{A})$. By Lemma 1, $(A) \Rightarrow(D)$. To show that (D) implies (C) assume that $P \subset \overline{\operatorname{Int} P}$ and that Int $P$ is connected and locally connected at every point of $P$. Let $B \subset \partial \Delta$ be a relatively open set, $F \subset B$ a relatively closed set of measure 0 and $f: F \rightarrow P$ a continuous function. In the case of noncompact $F$ write $F=\bigcup_{j=1}^{\infty} F_{j}$ where $F_{j}(j \in N)$ are nonempty compact subsets of $\Delta \cup B$ such that there exist disjoint open sets $0_{j} \subset \Delta \cup B$ $(j \in N)$ satisfying $F_{j} \subset 0_{j}(j \in N)$ ([8], see also Part 1 of the proof of Theorem 4). For each $j \in N, f_{j}=f \mid F_{j}$ is a continuous function from $F_{j}$ to $P$. Now Theorem 3 applies to show that by the properties of $P$ there exists for each $j \in N$ a homotopy $p_{j}: F_{j} \times I \rightarrow P$ satisfying $p_{j}(s, t) \in \operatorname{Int} P\left(s \in F_{j}, 0 \leqq t<1\right)$ and $p_{j}(s, 1)=f_{j}(s)\left(s \in F_{j}\right)$. Define $p: F \times I \rightarrow P$ by $p \mid F_{j} \times I=p_{j}(j \in N)$. By the properties of $p_{j}$ and $F_{j}(j \in N) p$ is continuous and satisfies $p(s, t) \in \operatorname{Int} P(s \in F$, $0 \leqq t<1)$ and $p(s, 1)=f(s)(s \in F)$. In the case when $F$ is compact the existence of such a $p$ is immediate by Theorem 3. Now by Theorem 4 there exists $\tilde{f} \in H_{B}(\Delta, X)$ satisfying $\tilde{f} \mid F=f$ and $\tilde{f}((\Delta \cup B)-F) \subset \operatorname{Int} P$. 
Proof of Theorem 2. Clearly $(\mathrm{C}) \Rightarrow(\mathrm{B}) \Rightarrow(\mathrm{A})$. It remains to prove that (A) implies (C). Assume that there exists a closed set $F \subset \partial \Delta$ of measure 0 with infinitely many points such that every continuous function $f: F \rightarrow P$ admits an extension $\tilde{f} \in A(\Delta)$ satisfying $\widetilde{f}(\bar{\Delta}) \subset P$.

Let $f: F \rightarrow P$ be a nonconstant continuous function. By the above assumption there exists a necessarily nonconstant extension $\tilde{f} \in A(\Delta)$ of $f$ satisfying $\tilde{f}(\bar{\Delta}) \subset P$. Since every nonconstant complexvalued analytic function is an open map [20] we have $\widetilde{f}(\Delta) \subset \operatorname{Int} P$. Consequently $(s, t) \mapsto p(s, t)=f(s t)$ is a continuous function from $F \times I$ to $P$ satisfying

$$
\begin{array}{ll}
p(s, t) \in \operatorname{Int} P & (s \in F, 0 \leqq t<1) \\
p(s, 1)=f(s) & (s \in F) .
\end{array}
$$

In the case when $f: F \rightarrow P$ is a constant, say $f(s)=x(s \in F)$ write $F=F_{1} \cup F_{2}$ where $F_{1}, F_{2}$ are disjoint compact sets. By the assumption $P$ consists of more than one point so that there is a $y \in P, \quad y \neq x$. Further, by the above assumptions there exist necessarily nonconstant functions $f_{1}, f_{2} \in A(\Delta)$ satisfying $f_{1}\left(F_{1}\right)=$ $f_{2}\left(F_{2}\right)=\{x\}, f_{1}\left(F_{2}\right)=f_{2}\left(F_{1}\right)=\{y\}$ and $f_{1}(\bar{\Delta}) \subset P, f_{2}(\bar{\Delta}) \subset P$. Now define $p: F \times I \rightarrow P$ as follows

$$
p(s, t)= \begin{cases}f_{1}(s t) & \left(s \in F_{1}, 0 \leqq t \leqq 1\right) \\ f_{2}(s t) & \left(s \in F_{2}, 0 \leqq t \leqq 1\right)\end{cases}
$$

Since $f_{1}$ and $f_{2}$ are open maps $p$ is a continuous function satisfying (5.1).

Having proved the existence of a continuous function $p: F \times I \rightarrow P$ satisfying (5.1) Theorem 4 applies to show that there exists $g \in A(\Delta, X)$ satisfying $g \mid F=f$ and $g(\bar{\Delta}-F) \subset \operatorname{Int} P$. Since $f$ was arbitrary it follows that (A) in Theorem 1 is satisfied for our $F$ and $P$. Consequently (C) follows by Theorem 1 .

3. Application and remarks, Given any set $P \subset C$ homeomorphic to $\bar{\Delta}$ there exists $f \in A(\Delta)$ satisfying $f(\bar{\Delta})=f(\partial \Delta)=P$. This is a consequence of Rudin's theorem. Below we present some generalizations of this result. The existence of $f \in A(\Delta)$ such that $f(\partial \Delta)$ fills some square had been proved before Rudin's theorem and had raised certain interest [16]. Church [5] gave a complete topological description of the sets $f(\partial \Delta), f \in A(\Delta)$.

Application 1. Given any nonempty compact set $P \subset C$ satisfying $P=\overline{\operatorname{Int} P}$ and such that Int $P$ is connected and locally connected at every point of $P$, there exists $\tilde{f} \in A(\Delta)$ satisfying $\tilde{f}(\bar{\Delta})=\tilde{f}(\partial \Delta)=P$. 
Proof. Let $F \subset \partial \Delta$ be a Cantor set of measure 0. By the compactness of $P$ there exists $f \in C(F)$ satisfying $f(F)=P$ [13]. Now by Corollary 1 there exists an extension $\tilde{f} \in A(\Delta)$ satisfying $f(\bar{d}) \subset P$.

Application 2. Given any nonempty set $P \subset C$ satisfying $P=$ $\overline{\text { Int } P}$ and such that Int $P$ is connected and locally connected at every point of $P$, there exists a continuous function $\widetilde{f}: \bar{A}-\{1\} \rightarrow C$, analytic on $\Delta$ and satisfying

$$
\widetilde{f}((\bar{\Delta}-\{1\}) \cap U)=\widetilde{f}((\partial \Delta-\{1\}) \cap U)=P
$$

for every neighbourhood $U \subset C$ of the point 1 .

Proof. Let $\left\{V_{n}: n \in N\right\}$ be a sequence of disjoint open ares in $\partial \Delta$ such that for every neighbourhood $U$ of the point 1 there exists $n_{U} \in N$ such that $V_{n} \subset U\left(n \in N, n>n_{U}\right)$. For each $n \in N$ let $F_{n} \subset V_{n}$ be a Cantor set of measure 0 . It is easy to construct a function $\alpha: N \rightarrow N$ satisfying

$$
N \subset \alpha(\{n, n+1, \cdots\}) \quad(n \in N) .
$$

Write $P=\bigcup_{n=1}^{\infty} P_{n}$ where $P_{n}$ are compacut sets. For each $n \in N$ there exists $f_{n} \in C\left(F_{n}\right)$ satisf ying $f_{n}\left(F_{n}\right)=P_{\alpha(n)}$ [13]. Put $F=\bigcup_{n=1}^{\infty} F_{n}$ and define $f: F \rightarrow P$ by $f \mid F_{n}=f_{n}(n \in N)$. Clearly $f$ is a continuous function. By Theorem 1 there exists a continuous extension $\widetilde{f}: \bar{\Delta}-$ $\{1\} \rightarrow X$, analytic on $\Delta$ and satisfying $\widetilde{f}(\bar{\Delta}-\{1\}) \subset P$. It is easy to see that $\tilde{f}$ has all the required properties.

The following application to vector-valued functions is perhaps more interesting. Its proof is the same as the proof of Application 1.

Application 3. Given any nonempty compact set $P$ in a finite dimensional complex normed space $X$ satisfying $P=\overline{\operatorname{Int} P}$ and such that Int $P$ is connected and locally connected at every point of $P$ there exists $\tilde{f} \in A(\Delta, X)$ such that $\widetilde{f}(\bar{\Delta})=\widetilde{f}(\partial \Delta)=P$.

REMARK. Note that in Application 2 we can replace $C$ by any finite-dimensional complex normed space.

Note that in the case when $X$ is infinite-dimensional one can not fill a subset of $X$ having nonempty interior with $f(\Delta \cup B)$ for some $f \in H_{B}(\Delta, X)$ since $f(\Delta \cup B)$ is always a countable union of compact subsets of $X$ and consequently its interior is empty. For the results about cluster sets in this case see [8,9]. 
Remark. If $X=C$ then by Theorem 2 a set $P \subset X$ containing more than one point has AEP if and only if it has PAEP. If $\operatorname{dim} X \geqq 2$ this is no longer true since there exist subsets $P$ of $X$ having AEP whose interior is empty. An example is $P=\overline{\Delta x}$ where $x \in X, x \neq 0$.

Problem. Give an example of a nonempty set $P \subset C^{2}$ satisfying $P=\overline{\text { Int } P}$ which has AEP but not PAEP.

REMARK. The results of the present paper give a partial solution to Problem 3 in [10].

ACKNowledgment. The author wishes to express his thanks to Professor Ivan Vidav and to professor Jože Vrabec for several very stimulating discussions while this paper was being prepared. A part of the results was obtained in November 1975 while the author was visiting Trinity College, Dublin. He wishes to express his thanks to Professor Trevor West and to Professor Richard Aron for organizing the visit and specially to Professor Aron for his hospitality.

\section{REFERENCES}

1. E. Bishop, A general Rudin-Carleson theorem, Proc. Amer. Math. Soc., 13 (1962), 140-143.

2. L. Brown, P. M. Gauthier and W. Hengartner, Continuous boundary behaviour for functions defined in the open unit disc, Nagoya Math. J., 57 (1975), 49-58.

3. L. Carleson, Representations of continuous functions, Math. Z., 66 (1957), 447-451. 4. P. T. Church, Global boundary behavior of meromorphic functions, Acta Math., 105 (1961), 49-62.

5. - Boundary images of meromorphic functions, Trans. Amer. Math. Soc., 110 (1964), 52-78.

6. E. F. Collingwood and A. J. Lohwater, The theory of cluster sets, Cambridge Tracts Math. Math. Phys. No. 56. Cambridge University Press, (1966).

7. J. Globevnik, The Rudin-Carleson theorem for vector-valued functions, Proc. Amer. Math. Soc., 53 (1975), 250-252.

8. - Analytic functions whose range is dense in a ball, J. Functional Analysis, 22 (1976), 32-38.

9. - The range of vector-valued analytic functions, Ark. Mat., 14 (1976), 113118. The range of vector-valued analytic functions II. Ark. Mat.; 14 (1976), 297-298.

10. On the range of analytic functions into a Banach space, To appear in Proc. Symp. Inf. Dim. Holomorphy, Campinas, (1975), Notas de Mat., North Holland.

11. - Analytic extensions of vector-valued functions, Pacific J. Math., 63 (1976), 389-395.

12. E. A. Heard and J. H. Wells, An interpolation problem for subalgebras of $H^{\circ}$. Pacific J. Math., 28 (1969), 543-553.

13. J. G. Hocking and G. S. Young, Topology, Addison-Wessley, 1961.

14. K. Hoffman, Banach Spaces of Analytic Functions, Prentice-Hall, 1962.

15. G. M. Leibowitz, Lectures on Complex Function Algebras, Scott, Foresman, 1970. 
16. G. R. MacLane, On the Peano curve associated with some conformal maps, Proc. Amer. Math. Soc., 6 (1955), 625-630.

17. M. H. A. Newman, Elements of the Topology of Plane Sets of Points, Cambridge Univ. Press, 1964.

18. D. M. Oberlin, Interpolation and vector-valued functions, J. Functional Analysis 15 (1974), 428-439.

19. W. Rudin, Boundary values of continuous analytic functions, Proc. Amer. Math. Soc., 7 (1956), 808-811.

20. - Real and Complex Analysis, McGraw-Hill, 1966.

21. E. L. Stout, On Some Restriction Algebras, Function Algebras, Ed. F. T. Birtel, Scott, Foresman, 1966, pp. 6-11.

22. - The Theory of Uniform Algebras, Bogden and Quigley, 1971.

23. G. T. Whyburn, Topological Analysis, Princeton University Press, 1964.

Received July 1, 1976 and in revised form October 27, 1976. This work was supported in part by the Boris Kidrič Fund, Ljubljana, Yugoslavia

UNIVERSITY OF LJUBLJANA

LJUBLJANA, YugosLavia 


\section{PACIFIC JOURNAL OF MATHEMATICS}

\section{EDITORS}

RICHARD ARENS (Managing Editor)

University of California

Los Angeles, CA 90024

R. A. BEAUMONT

University of Washington

Seattle, WA 98105

C. C. Moore

University of California

Berkeley, CA 94720

\section{J. DugundJI}

Department of Mathematics

University of Southern California

Los Angeles, CA 90007

R. Finn and J. Milgram

Stanford University

Stanford, CA 94305

\section{ASSOCIATE EDITORS}

\section{E. F. BECKENBACH}

B. H. NEUMANN

F. WoLF

K. YOSHIDA

\section{SUPPORTING INSTITUTIONS}

\author{
UNIVERSITY OF BRITISH COLUMBIA \\ CALIFORNIA INSTITUTE OF TECHNOLOGY \\ UNIVERSITY OF CALIFORNIA \\ MONTANA STATE UNIVERSITY \\ UNIVERSITY OF NEVADA \\ NEW MEXICO STATE UNIVERSITY \\ OREGON STATE UNIVERSITY \\ UNIVERSITY OF OREGON \\ OSAKA UNIVERSITY
}

\author{
UNIVERSITY OF SOUTHERN CALIFORNIA \\ STANFORD UNIVERSITY \\ UNIVERSITY OF HAWAII \\ UNIVERSITY OF TOKYO \\ UNIVERSITY OF UTAH \\ WASHINGTON STATE UNIVERSITY \\ UNIVERSITY OF WASHINGTON \\ AMERICAN MATHEMATICAL SOCIETY
}

The Supporting Institutions listed above contribute to the cost of publication of this Journal, but they are not owners or publishers and have no responsibility for its content or policies.

Mathematical papers intended for publication in the Pacific Jaurnal of Mathematics should be in typed form or offset-reproduced, (not dittoed), double spaced with large margins. Please do not use built up fractions in the text of your manuscript. You may however, use them in the displayed equations. Underline Greek letters in red, German in green, and script in blue. The first paragraph or two must be capable of being used separately as a synopsis of the entire paper. Items of the bibliography should not be cited there unless absolutely necessary, in which case they must be identified by author and Journal, rather than by item number. Manuscripts, in triplicate, may be sent to any one of the editors. Please classify according to the scheme of Math. Reviews, Index to Vol. 39. All other communications should be addressed to the managing editor, or Elaine Barth, University of California, Los Angeles, California, 90024.

The Pacific Journal of Mathematics expects the author's institution to pay page charges, and reserves the right to delay publication for nonpayment of charges in case of financial emergency

100 reprints are provided free for each article, only if page charges have been substantially paid. Additional copies may be obtained at cost in multiples of 50 .

The Pacific Journal of Mathematics is issued monthly as of January 1966. Regular subscription rate: $\$ 7200$ a year (6 Vols., 12 issues). Special rate: $\$ 36.00$ a year to individual members of supporting institutions.

Subscriptions, orders for back numbers, and changes of address should be sent to Pacific Journal of Mathematics, 103 Highland Boulevard, Berkeley, California, 94708.

PUBLISHED BY PACIFIC JOURNAL OF MATHEMATICS, A NON-PROFIT CORPORATION

Printed at Kokusai Bunken Insatsusha (International Academic Printing Co., Ltd.). 8-8, 3-chome, Takadanobaba, Shinjuku-ku, Tokyo 160, Japan.

Copyrit (C) 1975 by Pacific Journal of Mathematics Manufactured and first issued in Japan 


\section{Pacific Journal of Mathematics \\ Vol. 69, No. $2 \quad$ June, 1977}

Carol Alf and Thomas Alfonso O'Connor, Unimodality of the Lévy spectral

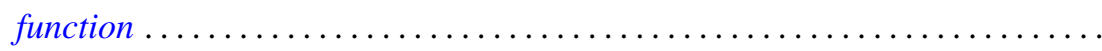

S. J. Bernau and Howard E. Lacey, Bicontractive projections and reordering of

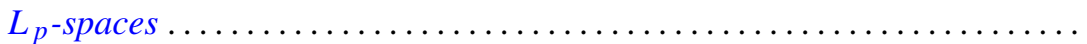

Andrew J. Berner, Products of compact spaces with bi-k and related spaces..... 303

Stephen Richard Bernfeld, The extendability and uniqueness of solutions of ordinary differential equations ...............................

Marilyn Breen, Decompositions for nonclosed planar m-convex sets ..........

Robert F. Brown, Cohomology of homomorphisms of Lie algebras and Lie

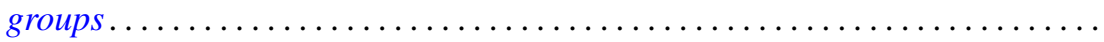

Jack Douglas Bryant and Thomas Francis McCabe, A note on Edelstein's iterative test and spaces of continuous functions ....................

Victor P. Camillo, Modules whose quotients have finite Goldie dimension ....... 333

David Downing and William A. Kirk, A generalization of Caristi's theorem with

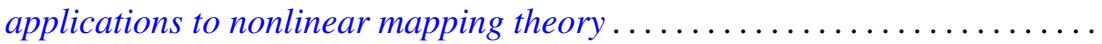

Daniel Reuven Farkas and Robert L. Snider, Noetherian fixed rings ...........

Alessandro Figà-Talamanca, Positive definite functions which vanish at

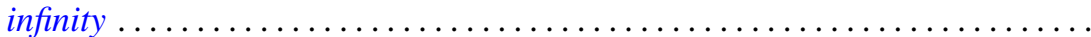

Josip Globevnik, The range of analytic extensions .................. 365

André Goldman, Mesures cylindriques, mesures vectorielles et questions de

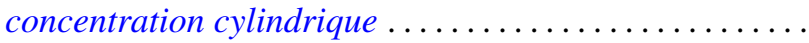

Richard Grassl, Multisectioned partitions of integers..........

Haruo Kitahara and Shinsuke Yorozu, A formula for the normal part of the

Laplace-Beltrami operator on the foliated manifold .... .

Marvin J. Kohn, Summability $R_{r}$ for double series .........

Charles Philip Lanski, Lie ideals and derivations in rings with involution ..

Solomon Leader, A topological characterization of Banach contractions . .

Daniel Francis Xavier O’Reilly, Cobordism classes of fiber bundles . .

James William Pendergrass, The Schur subgroup of the Brauer group . .

Howard Lewis Penn, Inner-outer factorization of functions whose Fourier series

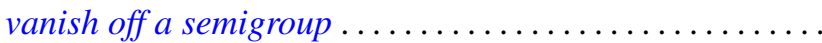

501

William T. Reid, Some results on the Floquet theory for disconjugate definite

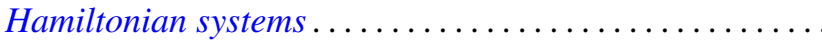

Caroll Vernon Riecke, Complementation in the lattice of convergence

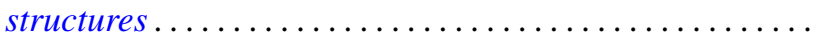

Louis Halle Rowen, Classes of rings torsion-free over their centers ......... 527

Manda Butchi Suryanarayana, A Sobolev space and a Darboux problem ....... 535

Charles Thomas Tucker, II, Riesz homomorphisms and positive linear maps.... 551

William W. Williams, Semigroups with identity on Peano continua ........... 557

Yukinobu Yajima, On spaces which have a closure-preserving cover by finite 